\title{
Multilinguales
}

Multilinguales

12 | 2020

Varia 2019

\section{Formes et valeurs du passif en tamazight}

Forms and values of the amazigh linguage passif

\section{Mustapha tidjet}

\section{OpenEdition}

Journals

Édition électronique

URL : https://journals.openedition.org/multilinguales/4483

DOI : $10.4000 /$ multilinguales.4483

ISSN : 2335-1853

\section{Éditeur}

Université Abderrahmane Mira - Bejaia

\section{Référence électronique}

Mustapha tidjet, « Formes et valeurs du passif en tamazight », Multilinguales [En ligne], 12 | 2020, mis en ligne le 14 février 2020, consulté le 30 juin 2021. URL : http://journals.openedition.org/ multilinguales/4483; DOI : https://doi.org/10.4000/multilinguales.4483

Ce document a été généré automatiquement le 30 juin 2021.

\section{(c) $)(1) \Theta$}

Multilinguales est mise à disposition selon les termes de la Licence Creative Commons Attribution Pas d'Utilisation Commerciale - Pas de Modification 4.0 International 


\section{Formes et valeurs du passif en tamazight $^{1}$}

Forms and values of the amazigh linguage passif

Mustapha tidjet

1 Les linguistes berbérisants répartissent en général la synthématique berbère en deux branches: une grande branche, représentant la tendance dominante, c'est la dérivation, qui se répartis elle-même en deux sous-branches: la dérivation grammaticale qui est très productive et les formations expressives qui sont d'une moindre productivité; une deuxième branche de moindre importance qui est la composition, elle est considérée traditionnellement comme étant marginale, à l'instar de Chaker (1991a : 179) qui affirme que

la composition n'est en berbère qu'un phénomène sporadique, peu productif, alors que la dérivation, tant verbale que nominale, constitue un système essentiel dans l'économie générale de la langue. La dérivation est le pivot, non seulement du lexique, mais aussi de la syntaxe de l'énoncé verbal.

Cependant, en synchronie, avec les créations néologiques et terminologiques, qui ne cessent de prendre de l'ampleur dans le lexique amazigh, cette affirmation doit être relativisée ${ }^{2}$.

2 Nous pouvons donc affirmer, sans grand risque d'erreur, que la dérivation et la composition sont les deux principaux procédés formels de la formation du lexique amazigh. A ces deux voies traditionnelles, nous ajouterons l'emprunt, signalé déjà par Mohand Akli Haddadou depuis 1985, comme une autre manière de combler les lacunes lexicales de la langue, notamment pour ce qui est du kabyle. Nous avons nous-mêmes (Tidjet, 1998 et 2013) essayé d'attirer l'attention des chercheurs sur les expressions figées qui sont devenues des objets investis par la recherche en lexicologie/ lexicographie depuis près d'un demi siècle, surtout dans les rangs des générativistes, et plus particulièrement dans le courant des linguistes qui s'intéressent à la traduction automatique des langues, mais elles restent malheureusement marginales dans le domaines des études amazighes. N'omettons pas de signaler quelques créations ex- 
nihilo d'unités néologiques, enrichissant par la même occasion les racines lexicales existantes.

Mais la dérivation grammaticale se taille la part du lion dans la formation du lexique amazigh. "C'est le procédé le plus vivant en synchronie, et surtout le plus apparent. Ayant attiré l'attention des chercheurs, dès le début des recherches dans le domaine berbère, il a été bien exploré. Les schèmes de dérivations sont assez bien établis, avec un paradigme fermé et restreint, et des valeurs sémantiques et fonctionnelles assez stables » (Tidjet, $2013: 45)$.

3 En théorie, à partir d'une racine consonantique (lexicale) indifférenciée, on peut obtenir, par affixation, un radical verbal, qui est une forme verbale simple associant la racine et, éventuellement, d'un affixe, et/ou un nominal ${ }^{3}$. De ceux-là, on peut obtenir des formes dérivées assez foisonnantes et des passages aisés entre les deux catégories. Cependant, en synchronie, on ne peut pas obtenir toutes les formes possibles pour chaque racine, le nombre de formes attestées étant souvent très restreint.

$\mathrm{Au}$ sein de la dérivation grammaticale elle-même, le verbe se caractérise par des latitudes combinatoires beaucoup plus régulières que le nom. En effet, à partir d'une forme verbale simple on peut obtenir une multitude d'autres formes verbales et même des noms (déverbatif concret, nom d'action verbale, nom d'agent, nom d'instrument). C'est-à-dire que les schèmes qui se combinent avec les verbes sont plus productifs que les autres.

Le verbe amazigh est composé d'une racine consonantique, d'une marque aspectuelle, d'un indice de personne et d'un schème de dérivation qui peut être zéro. Il est obtenu par préfixation d'un schème dérivationnel à un radical verbal et d'un schème verbalisateur à une racine nominale. C'est cette combinaison avec des schèmes qui est exploitée pour l'obtention des formes passives.

\section{Problématique}

4 Il est généralement admis que le passif amazigh est réalisé par trois formes : $\mathrm{mm}$-, ttwet le morphème zéro dans le cas des verbes mixtes. Il n'est souvent pas expliqué pourquoi trouvons-nous trois manières différentes d'exprimer une même réalité linguistique, ce qui est en contradiction flagrante avec la règle de l'économie linguistique valable pour toutes les langues naturelles.

Nous allons, dans le cadre de cette contribution, essayer d'y remédier en explicitant les valeurs sémantiques et fonctionnelles de chacune de ces formes.

Nous essayerons également de montrer l'émergence d'une quatrième forme. En effet, les dérivés en $n-/ n n$ - ont toujours étaient considérés comme des variantes phonétiquement conditionnées de la forme en $\mathrm{mm}$-, ce qui est certainement vrai au plan diachronique. Cependant, nous avons relevé en synchronie des combinaisons de ce morphème avec des verbes incompatibles avec le morphème du passif $\mathrm{mm}$-. Ces cas étant rarissimes, nous pensons qu'il s'agit du début d'apparition d'une nouvelle forme.

\section{Passif dans les verbes mixtes}

5 Ce sont des verbes qui, par une même forme, peuvent nous fournir des valeurs transitives et intransitives, qu'on ne peut distinguer qu'à travers le contexte linguistique dans lequel ils sont insérés. Si le verbe est suivi d'un nom à l'état 
d'annexion, c'est-à-dire que ce nom n'est qu'un complément explicatif de l'indice de personne, et qu'en plus il n'admet aucune autre extension directe, nous avons alors affaire à une valeur passive du verbe (et là nous pouvons parler d'un verbe passif). Par contre si le nom qui suit le verbe est à l'état libre, ce qui en fait un complément d'objet direct, qui est une caractéristique des verbes transitifs, alors ce verbe a la valeur d'un verbe actif-transitif. Exemples :

Le verbe qqen « attacher »; il est actif-transitif dans l'expression :

yeqgen azger "il a attaché un bœuf ",

mais il est passif-intransitif dans l'expression:

yeqqen uzger « il est attaché, le bœuf »;

dans cette deuxième expression le nom uzger n'est qu'un complément explicatif de l'indice de personne, ceci nous montre que l'action est subie par cet agent/sujet, ce qui est une caractéristique des voies passives ; d'un autre coté, nous ne pouvons lui ajouter de complément d'objet direct, ce qui caractérise encore les verbes intransitifs. Nous remarquons que la même forme yeqqen a la valeur de l'actif-transitif dans la première expression et celle du passif-intransitif dans la seconde.

Nous pouvons dire exactement la même chose des verbes comme :

Yebna axxam "il a construit une maison" > yebna uxxam "elle est construite, la maison»;

Yezger abrid « il a traversé la route » > yezger ubrid « la route est passée ».

L'utilisation d'un verbe mixte avec cette deuxième valeur peut être considérée comme étant une première forme du passif de la langue kabyle, et de la langue amazighe en général.

\section{Passif avec schème}

7 En plus de cette forme non-marquée, nous avons trois autres formes obtenues par préfixation de morphèmes du passif : $t$ tw- (et ses différentes variantes phonétiquement conditionnées : $t t u-, t t-), m m$ - et $n n$ - (ou sa variante $n$-).

Notons ici que beaucoup de spécialistes du domaine amazigh considèrent le morphème $n n$ - comme étant une variante phonétiquement conditionnée du morphème $\mathrm{mm}^{-4}$. Cependant, à moins de postuler l'existence de deux morphèmes nn- distincts, position qui est à mon avis difficilement défendable, les valeurs du $n n$ - ne se réduisent pas à celles du mm- (comme nous allons le voir infra, voir la rubrique : Le réfléchis).

La fonction de ces morphèmes est fondamentalement la même,

«ils transforment, dans la quasi-totalité des cas, un verbe transitif ou mixte en un verbe strictement intransitif dont le premier déterminant est le "patient" d'un procès qui lui est extérieur » (Chaker, 1991a : 193).

\subsection{Verbes transitifs}

8 Un verbe transitif peut acquérir la valeur du passif s'il est rattaché à l'un des morphèmes cités précédemment et devient strictement intransitif et, par conséquent, n'admet plus de complément d'objet direct. Les noms qui succèdent directement à ces verbes sont dit compléments explicatifs parce qu'ils se contentent d'expliciter l'agent ou le patient qui est déjà annoncé par l'indice de personne. Ils sont, quand leur 
morphologie le permet, à l'état d'annexion. D'autres syntagmes nominaux peuvent leur être rattachés mais seulement par l'entremise d'une préposition.

Verbe transitif + morphème du passif > dérivé intransitif

ečč « manger » > ttwačč, mmečč « être mangé »

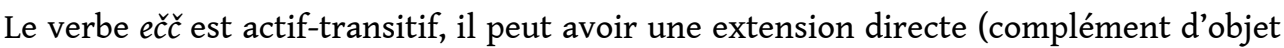
direct), c'est-à-dire qu'un nom à l'état libre peut lui être directement rattaché en plus, bien sur du complément explicatif qui, lui, est compatible avec tous les types de verbes. Nous pouvons alors former un syntagme tel que : yečča uqcic tateffaht « l'enfant à mangé une pomme». Par contre les formes passives sont strictement intransitives. Nous pouvons citer beaucoup d'autres exemples de ce type de verbes :

Aker « voler » > ttwiker « être volé »

$E f k$ « donner » > nnefk « être donné »

Bder « citer » > ttwibder « être cité »

Bges « ceindre » > ttwibges « être ceint "

Bren « retourner » > nnebran « être retourné ; se retourner »

Bru « divorcer, répudier » > nnebru « être divorcé, être répudié ».

Ddem « prendre » > ttwiddem « être pris »

\subsection{Verbes mixtes}

10 Ce sont des verbes qui peuvent, sans changer de formes, avoir l'une des deux valeurs active ou passive. Ces verbes, contrairement aux précédents, ne peuvent se combiner qu'avec le morphème $t t w$-, les morphèmes $m m$ - et $n n$ - ne peuvent leur être rattachés.

Verbe mixte $+t t w$ - > dérivé intr.

qqen " attacher/être attaché » > ttwaqqen " être attaché »

kteb/aru " écrire/être écrit » > ttwakteb/ttwaru ${ }^{5}$ "être écrit "

mdel « enterrer/être enterré » > ttwamdel « être enterré »

Certains verbes mixtes ne se combinent même pas avec morphème, se contentant des deux valeurs active et passive exprimées par une même forme :

Cuff « gonfler, être gonflé ; enfler »

\section{Valeurs et opposition des morphèmes du passif}

11 S'il est aisé de déterminer leur fonction syntaxique, leur valeur sémantique est plus difficile à saisir ; cependant leurs combinaisons avec les verbes mixtes et intransitifs ayant, à la base, cette caractéristique d'intransitivité et la possibilité de combiner les deux morphèmes $m m$ - ou $n n$ - et $t t w$ - à un même radical nous permettent de préciser, quelque peu, cette valeur :

L'idée d'une opposition actif (non-marqué) passif (marqué), ou vis-versa, doit être d'emblée évacuée, car un verbe simple (sans changer de forme) peut donner les deux valeurs (active et passive);

Yeqqen azger $\sim$ yeqqen uzger

« il a attaché un bœuf » «il est attaché, le bœuf »

Tura taktubt $\sim$ tura tektubt

« elle a écrit un livre » « le livre est écrit », littéralement « elle est écrite le livre ${ }^{6}$ » 
12 Nous remarquons ici que les valeurs du passif («il est attaché » et « elle est écrite ») sont rendues par les mêmes formes verbales qui sont à l'origine des valeurs actives. La même forme yeqqen est tantôt un verbe actif, tantôt un verbe passif. En fait, c'est la morphologie du nom qui décide de la valeur, active ou passive, du verbe. Si le nom est à l'état libre, caractérisé par une forme non marquée, ce qui lui confère la fonction de complément d'objet direct, alors le verbe est obligatoirement actif. Par contre si le nom est à l'état d'annexion, ce qui est indiqué par une forme particulière, il acquière par conséquent la fonction de complément explicatif de l'indice de personne, dans ce cas c'est le passif qui est exprimé par le verbe.

Deux constructions passives, l'une marquée l'autre non-marquée, sont possibles pour un même verbe, avec les verbes dits « mixtes »;

Yeqqen uzger yettwaqqen uzger

« il est attaché le bœuf » «il a été attaché le bœuf »

« et, l'hypothèse d'une parfaite synonymie entre les deux types d'énoncés doit

d'emblée être exclue comme radicalement contraire au principe général

d'économie qui régit toute langue » (Chaker, 1991a : 190);

Deux morphèmes peuvent être attachés à un même radical ;

ěčc « manger » > mmečč, ttwačč « être mangé »

Il arrive même que les deux formes soient conjointement rattachées à une même forme verbale simple :

efk « donner » > nnefk « être donné », ttunefk « être donné »

Ainsi nous comprenons qu'il y a une opposition claire et nette entre le morphème ttwd'une part et les morphèmes $\mathrm{mm}$ et $n n$ - d'autre part, leurs valeurs ne peuvent par conséquent être confondues.

D'autre part, on remarquera que $\mathrm{mm}$ - et $n n$ - ne sont jamais combinés au même radical, et le sont, généralement, avec les verbes potentiellement transitifs, qui n'ont pas en base la valeur de passif. On peut alors parler d'une triple opposition : (1) actif ; (2) passif avec $t$ tw- ; et (3) passif avec $\mathrm{mm}$-, nn- et le morphème zéro des verbes mixtes.

Il semblerait que ces trois dernières formes nous donnent des verbes avec lesquels l'agent est totalement absent, son existence n'est même pas envisagée ou, à la limite, complètement évacué, alors qu'avec le $t$ tw-, même en cas d'absence, son existence est tout de même posée. On peut même avoir des expressions où il est explicitement cité : yettwawet s yimawlan-is « ce sont ses parents qui l'ont brisé ».

\section{Le réfléchis}

14 Le morphème nn-, combiné avec certains verbes actifs, peut nous donner des verbes réfléchis :

Bren « tordre " > nnebran " se tordre "

Qleb « tourner » > nneqlab « se retourner »

Dans des cas pareils, on ne peut pas distinguer le réfléchis des valeurs du passif sans recourir au contexte qui précise la signification du verbe. Par contre avec d'autres verbes, comme efk, la valeur du passif est clairement établie. Cette possibilité d'avoir ou nom des sémantismes différents, selon le verbe auquel il est combiné, le distingue nettement du morphème $\mathrm{mm}$ - 


\section{Un ou deux verbes?}

15 Un certain nombre de verbes polysémiques pose un problème de classification. Ils sont généralement mis dans la catégorie des verbes mixtes. Mais à regarder de plus prêt leur fonctionnement, on se rendra vite compte que leurs comportements changent selon le sens dans lequel ils sont pris.

Fsi est un verbe polysémique, il a les sens de « délier, dénouer » et de «fondre ». Avec le premier sens nous obtenons un verbe mixte qui peut nous donner l'actif plus le passif à l'aide une seule et même forme :

Tefsi takerrust « elle a dénoué un nœud » et tefsi tkerrust " elle est dénoué, nœud ${ }^{7}$ » Par contre avec la deuxième signification nous ne pouvons obtenir que la valeur $\mathrm{du}$ passif :

Yefsi udfel « il est fondu, neige ${ }^{8}$ "

Pour avoir la voie active nous devons avoir recours au morphème s- de l'actif/transitif :

Yessefsi adfel « il a fait fondre la neige »;

Nous sommes en présence d'une situation différente avec le verbe ali, ce verbe ne s'utilise qu'avec la valeur active-transitive quand il signifie «monter, escalader, grimper»:

Yuli ayrab « il a escaladé un mur »

Et seulement comme passif avec le sens de bâtir/construire :

Yuli uxxam « elle est bâtie/construite la maison »

On doit encore recourir au morphème $s$ - pour avoir la valeur active tout en conservant sa signification :

Yessuli axxam « il a bâtie une maison »

16 Nous avons une troisième situation avec le verbe herrek. En effet ce verbe a des significations et des fonctionnements complètement différents en passant d'un premier déterminant (complément explicatif) non-humain à un premier déterminant humain. Dans la première situation nous avons un verbe mixte avec le sens de «bouger »:

Therrek tezrut « elle (la pierre) a bougé »

Iherrek uzrem « il s'est déplacé, le serpent »

Iherrek uzrem taqerruyt-is " il a remué, le serpent, sa tête "

Dans le second cas, la signification change selon la présence ou l'absence d'un second déterminant (complément d'objet direct)

Therrek taz̧rut « elle a déplacé la pierre »

Therrek tmetțut tazrut « elle a déplacé, la femme, la pierre », «la femme a déplacé la pierre, elle l'a faite bougé »;

Par contre si l'expression (verbe + complément explicatif) n'est pas complétée par un deuxième déterminant, on aura une valeur passive, sans possibilité d'une formation active avec le morphème $s^{-}$, mais avec le sens de " être dérangé, être fou » :

Iherrek urgaz « il est dérangé/fou, homme».

\section{Vers de nouvelles valeurs}

17 Les verbes intransitifs, qui sont passifs à la base, n'acceptent pas de combinaisons avec les morphèmes du passif. C'est le cas par exemple avec les verbes comme $\gamma l i$ « être tombé ; s'effondrer ", rib «s'effondrer, s'écrouler; s'affaisser ». Les seules formes que nous pouvons obtenir au prétérit 3è personne masculin singulier sont yeyli, irab; yeyli 
uqcic « il est tombé, le garçon », yeyli urrab « il s'est effondré, le mur », irab ukeddar, lhid « il s'est affaissé, le mur ».

Irid « être lavé » est un verbe intransitif qui ne devrait normalement pas accepter de se combiner avec les morphèmes du passif, cependant nous avons rencontré la forme nnired dans l'expression ad tennirded $d^{9}$ " que tu sois lavé », c'est une forme d'imprécation pour souhaiter la mort à quelqu'un ${ }^{10}$.

18 Des formes dérivées peuvent acquérir des acceptions qui n'ont aucune relation de sens direct avec le verbe simple. Nous prenons le verbe qleb qui signifie « tourner, retourner, renverser ", il peut prendre le sens prévisible de "se retourner, se renverser ", mais il peut également signifier « se détracter, changer d'avis » qui est impossible à prévoir à partir des sens de base du verbe simple et du schème de dérivation. Nous pouvons également cité rnu « ajouter » et nnernu " grandir ».

Les préfixes $m m$ - et $n n$ - sont en complémentarité avec le morphème « zéro » des verbes mixtes. Ils forment, tous les trois, un type de verbes qu'on peut qualifier de prédicats d'existence (cf. Chaker, 1991b : 190 et L. Galand, 1977 : 295) qui entretiennent avec leurs indices personnels une relation non spécifiée (« agent », « patient », « attributaire »). Ils réfèrent plus à l'état (résultat d'un procès) qu'au procès lui-même.

19 Avec la marque $t t w$ - l'indice de personne est clairement posé comme patient, l'agent, même absent, est envisagé. Avec les autres formes, la relation qui existe entre le premier déterminant et le procès n'est pas spécifiée, néanmoins ce déterminant est plus proche d'un patient que d'un agent : ibna urrab « le mur est construit (parce qu'il a été construit)» (Galand, 1977 : 295), ad ibnu uðrab-a « ce mur se construira (parce qu'on va le construire) ", on remarquera que dans ces deux exemples on a affaire à des affirmations, et surtout que l'accent est mis d'une part, sur l'état du premier déterminant (après accomplissement du procès), d'autre part sur le résultat du procès (donc du prédicat), le procès lui-même n'est même pas envisagé d'où l'absence totale de l'agent.

\section{BIBLIOGRAPHIE}

Si au plan fonctionnel, nous pouvons nous contenter des remarques et conclusions globales des berbérisants que nous avons cités tout le long de cette modeste contribution, sur le plan sémantique par contre il nous faut reprendre l'étude de ces schèmes pour en faire des investigations systématiques et plus pointues afin de déterminer les valeurs exactes de chacun des morphèmes. Comme indiqué plus haut, le morphème nn- est très polysémique il est à distinguer du mm- qui reste, semble-t-il, une simple forme du passif.

ACHAB, R., « Problèmes de néologie berbère, Remarques sur l'"amawal" ", in Etudes et documents berbère 8, EDISUD, 1991, pp. 97-111.

ACHAB, R., La Néologie Lexicale Berbère : Approche critique et propositions, Thèse de doctorat (nouveau régime), INALCO, Paris, 1994. 
BASSET, A., La langue berbère, Publisched for : International African Institute, By the : Oxford University Press, London, New York, Toronto, 1952.

BENLAKHDAR, M., « Passif ou moyen : cherchez l'agent ! ", in Etudes et Documents Berbères 14, EDISUD, 1996, pp. 213-222.

CHAKER, S., Manuel de Linguistique Berbère I, éd. Bouchène, Alger, 1991a.

CHAKER, S., "Eléments de prosodie berbère, Quelques données exploratoires ", in Etudes et documents berbère 8, EDISUD, 1991b, pp. 5-25.

CHAKER, S., Linguistique Berbère :études de syntaxe et de diachronie, éd. PETEERS ; Paris-Louvain, 1995.

CHAKER, S., « Diathèse (voix) », in encyclopédie Berbère XV, EDISUD, Aix-en-Provence, France, 1995b, pp. 2297-2303.

GALAND, L., «Continuité et Renouvellement d'un Système Verbale : le Cas du Berbère », in Bulletin de la Société de Linguistique de Paris, Tome LXXII, Fascicule 1, 1977, pp. 275-303.

HADDADOU, M.A., Structures Lexicales et Significations en Berbère (Kabyle), Thèse de Doctorat 3ème cycle,ș Aix en Provence, 1985.

TIDJET, M., Polysémie et abstraction dans le lexique amazigh (kabyle), Magister, Département de Langue et Culture Amazighes, université A. Mira, Bejaia, 1998.

TIDJET, M., « La langue comme facteur de résistance identitaire : traitement des emprunts en Kabylie » in Awal 34, éditions de la Maison des Sciences de l'Homme, Paris, 2006, pp. 33-60.

TIDJET, M., La patronymie dans les daïras de Timezrit, Sidi-Aich et Chemini : étude morphologique et sémantique, thèse de Doctorat es sciences, université M. Mammeri de Tizi Ouzou, 2013.

\section{NOTES}

1. Cette contribution est bâtie essentiellement sur la variante kabyle.

2. A notre connaissance, il n'y a pas de travaux récents qui tiennent compte de ces phénomènes nouveaux pour connaître la réalité actuelle. Les règles de la syntématique amazighe sont globalement respectées (cf. Tidjet 1998 et 2006 ; Achab, 1994), mais il semble que les proportions relatives entre les deux tendances ne soient pas les mêmes, la composition est plus présente qu'elle ne l'est dans le langage commun (constat purement empirique de notre part).

3. «Le lexème berbère est constitué d'une racine consonantique est d'un schème formant qui permet son actualisation. A la même racine on peut adjoindre un schème verbal (caractérisé par un indice de personne est une marque aspectuelle) pour obtenir un verbe ou un schème nominal (caractérisé par le genre, le nombre et l'état) pour obtenir un nom » (Tidjet, 2013 : 43).

4. «La forme (très rare) $n$ - n'est qu'une variante phonétiquement conditionnée de m(dissimilation devant radical verbal comportant une autre labiale : $f k$ "donner" $+m-=m$ - $f k>n$ $f k »$ (Chaker, 1995b : 2298)

5. Nous remarquons ici que l'emprunt à l'arabe kteb a hérité des latitudes combinatoires du verbe amazigh aru qu'il a supplanté, bien sûr en conservant certains sémantismes que le premier n'en a pas, surtout ce qui est relatif au religieux porté par le nom lmektoub « la destinée ».

6. Il faut tenir compte que taktubt «livre» est en kabyle un nom féminin, d'où le pronom personnel « elle».

7. Nœud est un nom féminin en berbère takerrsut.

8. Neige étant masculin en berbère. 
9. Expression que nous avons recueillie dans la région d'At Mlikech, Mlle Oulebsir Fadila, une enseignante au DLCA de Bejaia habitant Tazmalt, que nous tenons ici à remercier.

10. Dans la pratique musulmane, les morts sont toujours lavés avant qu'ils ne soient enterrés, d'où cette expression.

\section{RÉSUMÉS}

Il est généralement admis que le passif amazigh est réalisé par trois formes : $\mathrm{mm}-$, $\mathrm{ttw}$ - et le morphème zéro dans le cas des verbes mixtes. Il n'est souvent pas expliqué pourquoi trouvonsnous trois manières différentes d'exprimer une même réalité linguistique, ce qui est en contradiction flagrante avec la règle de l'économie linguistique valable pour toutes les langues naturelles. Nous allons, dans le cadre de cette contribution, essayer d'y remédier en explicitant les valeurs sémantiques et fonctionnelles de chacune de ces formes. Nous essayerons également de montrer l'émergence d'une quatrième forme. En effet, les dérivés en n-/nn- ont toujours étaient considérés comme des variantes phonétiquement conditionnées de la forme en $\mathrm{mm}-$, ce qui est certainement vrais au plan diachronique. Cependant, nous avons relevé en synchronie des combinaisons de ce morphème avec des verbes incompatibles avec le morphème du passif mm-. Ces cas étant rarissimes, nous pensons qu'il s'agit du début d'apparition d'une nouvelle forme.

It is generally accepted that the Amazigh passive is realized by three forms : $\mathrm{mm}_{-}^{-}, \mathrm{ttw}$ - and the zero morpheme in the case of mixed verbs. It is often not explained why we find three different ways of expressing the same linguistic reality, which is in flagrant contradiction with the rule of linguistic economy valid for all natural languages. We will, within the framework of this contribution, try to remedy it by explaining the semantic and functional values of each of these forms. We will also try to show the emergence of a fourth form. Indeed, derivatives in n-/nnhave always been considered as phonetically conditioned variants of the form in mm-, which is certainly true on the diachronic level. However, in synchrony, we have noted combinations of this morpheme with verbs incompatible with the passive morpheme mm-. These cases being extremely rare, we think that it's about the beginning of appearance of a new form.

\section{INDEX}

Mots-clés : études amazighes, kabyle, morphologie, sémantique, syntaxe

Keywords : amazigh studies, kabyle, morphology, semantics, syntax

\section{AUTEUR}

MUSTAPHA TIDJET

Centre de Recherche en Langue et Culture Amazighes (CRLCA), Bejaia, Algérie. 